\title{
Programas de Intervenção na Adolescência: Considerações sobre o Desenvolvimento Positivo do Jovem
}

\author{
Gisele de Rezende Franco ${ }^{1}$ \\ Marisa Cosenza Rodrigues \\ Departamento de Psicologia da Universidade Federal de Juiz de Fora, Juiz de Fora, \\ Minas Gerais, Brasil
}

\begin{abstract}
Resumo
O presente artigo tem como objetivo discutir fundamentos teórico-conceituais e empíricos que embasam programas preventivos e de promoção em saúde mental na adolescência, com enfoque no desenvolvimento positivo. Constata-se que o Brasil apresenta progressos no que tange ao desenvolvimento e implementação de programas interventivos tendo avançado no campo da promoção de competências e do bem-estar dos jovens, ampliando sua ação para além da prevenção de comportamentos de risco. São tecidas considerações sobre contribuições da psicologia positiva para os programas interventivos frente à necessidade de reavaliar potencialidades e virtudes humanas. São também destacados os avanços nos estudos empíricos da adolescência, apontando as tendências da ciência e a emergência de investigações oriundas da aplicação das teorias sob a visão positiva do jovem. Por fim, apresenta-se uma breve contextualização dos programas nacionais com foco na psicologia positiva. Conclui-se pela pertinência e interesse do desenvolvimento de intervenções baseadas nesta vertente teórico-prática, ressalta-se contudo, a necessidade de buscar o desenvolvimento positivo do jovem de modo efetivo nos programas interventivos nacionais, tal como ocorre no cenário internacional.
\end{abstract}

Palavras-chave: Pesquisa de intervenção, prevenção, promoção, adolescência, desenvolvimento positivo.

\section{Adolescent Intervention Programs: Considerations on Positive Youth Development}

\begin{abstract}
This article aims to discuss theoretical, conceptual and empirical foundations that support prevention and promotion programs in mental health in adolescence, focusing on the positive development. It appears that Brazil exhibits progress regarding the development and implementation of interventional programs, having advanced in the field of promoting skills and well-being of young people, and expanding its reach beyond the prevention of risk behaviors. The paper makes considerations on the contributions of positive psychology to interventional programs regarding the need to reassess potential and human virtues. It also highlights the advances in empirical studies of adolescence that indicate trends in science, and the emergence of investigations arising from the application of the young's positive outlook theories. Finally, we briefly discuss the background of the national programs focused on positive psychology. The paper agrees with the pertinence and interest of the development of interventions based on this theoretical and practical approach, we emphasize however, the need to
\end{abstract}

Endereço para correspondência: Rua Doutor Moacir de Castro Xavier, 54, Bairro Linhares, Juiz de Fora, MG, Brasil 36060-490. E-mail: gisele.franco2008@hotmail.com e rodriguesma@terra.com.br Apoio Financeiro: Bolsista UFJF-Monitoria. 
seek the positive youth development effectively in national interventional programs, such as occurs in the international scene.

Keywords: Intervention research, prevention, promotion, adolescence, positive development.

\section{Programas de Intervención en la Adolescencia: Consideraciones sobre el Desarrollo Positivo de la Juventud}

\section{Resumen}

Este artículo tiene como objetivo discutir los fundamentos teóricos, conceptuales y empíricas que apoyan los programas de prevención y promoción de la salud mental en la adolescencia, centrándose en el desarrollo positivo. Parece que Brasil exhibe los avances en cuanto a la elaboración y aplicación de programas de intervención, después de haber avanzado en el campo de la promoción de las habilidades y el bienestar de los jóvenes, y ampliar su alcance más allá de la prevención de conductas de riesgo. En el artículo se hace consideraciones sobre las contribuciones de la psicología positiva a los programas de intervención con respecto a la necesidad de reevaluar las virtudes posibles y humanos. También se destacan los avances en los estudios empíricos sobre la adolescencia que indican las tendencias de la ciencia, y la aparición de las investigaciones derivadas de la aplicación de las teorías de las perspectivas positivas de la juventud. Finalmente, se discuten brevemente los antecedentes de los programas nacionales centrados en la psicología positiva. En el artículo está de acuerdo con la pertinencia y el interés del desarrollo de las intervenciones basadas en este enfoque teórico y práctico, insistimos sin embargo, la necesidad de buscar el desarrollo positivo de los jóvenes de manera efectiva en los programas de intervención nacionales, como ocurre en la escena internacional.

Palabras clave: Investigación intervención, prevención, promoción, adolescencia, desarrollo positivo.

Sob a visão contemporânea da adolescência, programas proativos implementados em diferentes contextos devem focalizar estratégias que priorizem o incremento de fatores de proteção, pois tendem a produzir efeitos benéficos, como a minimização de problemas emocionais, sociais e comportamentais (Catalano, Berglund, Ryan, Lonczak, \& Hawkins, 2004; Murta, 2007). De modo específico, há evidências de efeitos significativos de intervenções promotoras do desenvolvimento de habilidades psicossociais (Cardozo, Dubini, Fantino, \& Ardiles, 2011). Essas evidências caminham em direção às contribuições da psicologia positiva, com vistas à promoção do desenvolvimento positivo do jovem, englobando a saúde de modo integral e investindo sobre o bem-estar e a qualidade de vida.

Nesse âmbito, assume relevância o movimento da psicologia positiva com enfoque nos aspectos potencialmente sadios dos indivíduos, devido ao seu interesse no fortalecimento e construção de competências pessoais. Entretanto, no
Brasil, apenas recentemente esses aspectos passaram a ser aplicados aos estudos sobre a adolescência (Gorayeb, Netto, \& Bugliani, 2003; Minto, 2005; Silva \& Murta, 2009). Nota-se um interesse crescente sobre a investigação na temática do desenvolvimento da resiliência, bem-estar e competências psicossociais, justificando a realização de estudos de cunho teórico, como é o caso do presente trabalho.

Para Catalano et al. (2004), a inclusão da abordagem do desenvolvimento positivo no campo da prevenção ampliou o foco das pesquisas na área e inseriu a promoção de competência nas intervenções. No que tange à adolescência, esta fase é marcada por inúmeras mudanças biológicas, cognitivas e socioemocionais (Santrock, 2014), que, muitas vezes, são acompanhadas por eventos prejudiciais a saúde, como o abuso de drogas, início precoce da sexualidade e o envolvimento em atos violentos e criminosos, dentre outros aspectos que afetam sobremaneira o jovem nesse período do desenvolvimento 
(Amparo, Galvão, Alves, Cardenas, \& Koller, 2008; Brasil, Alves, Amparo, \& Frajorge, 2006; De Antoni \& Koller, 2000; Heilborn et al., 2002; Marques \& Cruz, 2000; Pesce, Assis, Santos, \& Oliveira, 2005; Yunes, 2003).

Nesse contexto é relevante considerar os recursos e as mudanças pertinentes aos adolescentes nas capacidades e habilidades pessoais. Para esse fim, serão destacados no presente estudo programas fundamentados em evidências e na perspectiva de que a construção de saúde e competências do bem-estar podem prevenir sérios e dispendiosos problemas para o adolescente, alinhando-se aos pressupostos da psicologia positiva focalizada na investigação de potencialidades e virtudes humanas.

Segundo Seligman (2011), a intervenção positiva tem a tarefa de possibilitar o aprendizado das pessoas para lidar com o sofrimento e funcionar bem, mesmo na presença de sintomas, mediante a construção de condições propícias para as pessoas florescerem, como por exemplo: ter emoção positiva, engajamento, sentido, realização e melhores relações humanas. Nesse enfoque, ao investir na adolescência a partir do incremento de competências sociais, emocionais e cognitivas pode-se proporcionar a maximização das potencialidades humanas e promover o florescimento saudável dos jovens, em direção à superação de eventuais comportamentos de risco (J. V. Lerner, Phelps, Forman, \& Bowers, 2009).

A partir dessas considerações, objetiva-se discutir aqui, fundamentos teóricos e empíricos que embasam programas preventivos e de promoção em saúde mental na adolescência, com foco no desenvolvimento positivo. Para tanto, este estudo divide-se em três seções. Na primeira são destacadas algumas contribuições teóricas da psicologia positiva para os programas interventivos que almejam fomentar o desenvolvimento positivo do jovem. Tendo em vista que a psicologia positiva está em pleno processo de solidificação dentro da ciência psicológica, uma reavaliação das potencialidades e virtudes humanas torna-se fundamental para a compreensão dos fatores protetores que contribuem para o desenvolvimento saudável nesta fase. $\mathrm{Na}$ segunda seção, apontam-se os avanços nos estudos empíricos da adolescência sendo destacadas as tendências da ciência em direção à aplicação das teorias com ideário positivo. Por último, faz-se uma breve contextualização das pesquisas nacionais com foco em aspectos contribuintes da psicologia positiva.

\section{Programas Preventivos: Contribuições da Psicologia Positiva}

Historicamente, diversas teorias ou visões sobre a adolescência foram construídas sob princípios organísmicos ou contextualistas, diferenciando-se em teorias biológicas, psicanalíticas, socioculturais e cognitivas, de acordo com os principais autores da época (Erik Erikson, 1968/1976; G. Stanley Hall, 1904; Inhelder \& Jean Piaget, 1958/1976; Sigmund Freud, 1856-1939; Margaret Mead, 1928/1979; Lev Vygotsky, 1896-1934). Entretanto, segundo Santrock (2014), mudanças importantes podem ser constatadas no modo como se caracteriza a atual geração de adolescentes, culminando em uma visão mais positiva desse período do ciclo vital.

No século atual, com os avanços dos estudos teóricos envolvendo o tema adolescência (Goosens, 2006; R. M. Lerner \& Steinberg, 2009) e a adoção de modelos sistêmicos (Bronfenbrenner, 1979/1996) para a compreensão de fenômenos do desenvolvimento, esse período passou a ser demarcado por fatores inter-relacionados de ordem individual, histórica e cultural. A partir desta visão relacional, os trabalhos referentes ao desenvolvimento do adolescente chegaram a estabelecer relações entre os aspectos individuais e contextuais, e a identificar potencialidades e tendências da ciência em direção a uma nova fase caracterizada pela aplicação das teorias e, por uma visão de adolescência saudável e/ou positiva (Santrock, 2014; Senna \& Dessen, 2012).

As teorias do desenvolvimento, como a teoria do apego (Bowlby, 1982), a teoria da aprendizagem social (Bandura, 1977) e a teoria da identidade (Erikson, 1968/1976) identificam tarefas, desafios e competências requeridas durante a adolescência, fornecendo os alicerces para a construção da abordagem do desenvolvimento 
positivo dos jovens que, por definição, refere-se ao "envolvimento em comportamentos pró-sociais e o evitamento de comportamentos que comprometem e prejudicam a saúde e o futuro" (R. M. Lerner \& Galambos, 1998, p. 435). Essa abordagem é citada por muitos autores (Benson et al., 2006; Catalano et al., 2004; Damon, 2004), entretanto, existem diferentes visões na literatura sobre a mesma, constatando-se uma referência maior sobre a construção de competências (cognitivas, sociais e emocionais).

A partir dessas teorias, Santrock (2014) afirma que o "desenvolvimento positivo dos jovens" (DPJ) também pode ser sustentado pelo movimento da psicologia positiva. Entretanto, apenas recentemente esse movimento foi reconhecido no Brasil, afinando-se com os achados de Paludo e Koller (2007) os quais indicam a escassez de trabalhos sobre os aspectos saudáveis mantidos durante o desenvolvimento.

Para Sheldon e King (2001), a psicologia positiva é o estudo científico das forças e virtudes próprias do indivíduo. No entanto, é importante destacar que esta perspectiva teórica foi precedida por vários autores e por temas de investigação que ilustram sua trajetória histórica. Gable e Haidt (2005) destacam os escritos de Willian James em 1902 sobre saúde mental, passando pelo interesse de Allport nas características humanas positivas em 1958. Em seguida, em 1968, os estudos de Maslow sobre as pessoas saudáveis e, as pesquisas de Cowan sobre resiliência em crianças e adolescentes em 2000.

Neste âmbito, o estudo das potencialidades e virtudes humanas pela psicologia positiva demonstra-se alinhado à abordagem do desenvolvimento positivo, que segundo J. V. Lerner et al. (2009), destaca os pontos fortes e as qualidades dos jovens, bem como maneiras de alinhá-los com recursos e suporte do ambiente (família, escola, grupo de pares, comunidade). Para os referidos autores, os jovens precisam ter acesso a contextos sociais positivos por meio de programas organizados especificamente para eles, e também ter pessoas competentes - como professores, líderes comunitários e mentores que possam capacitá-los com relação aos valores associados à saúde e ao bem-estar comum.
Segundo Moore e Keyes (2003), do ponto de vista da psicologia positiva, tem-se buscado ultrapassar uma visão negativa e deficitária que predominou na Psicologia, por influência do modelo biomédico, que priorizava a prevenção dos resultados negativos sobre o desenvolvimento humano ao contrário da promoção de desenvolvimento positivo.

De acordo com Seligman (2011), a psicologia positiva resgata o caráter preventivo ao empreender avanços em estudos e pesquisas sobre a prevenção numa perspectiva mais focada na construção de competências, e não na correção de fragilidades. Como referido pelo autor, essa corrente está alicerçada sobre cinco pilares, a saber: o estudo da emoção positiva (felicidade e satisfação com a vida), do engajamento (envolvimento), do sentido (significado), das realizações positivas (conquistas) e dos bons relacionamentos.

Para tanto, segundo Seligman e Csikszenmihalyi (2000), busca-se privilegiar a necessidade de estudos e intervenções sustentadas nas construções subjetivas que envolvem felicidade, otimismo, companheirismo, autoestima, autoconceito, bem-estar, habilidades interpessoais indicativas da vida saudável e resiliência. Especificamente, a resiliência é definida como um processo que atua na presença de risco para produzir resultados positivos no desenvolvimento (Poletto \& Koller, 2006).

Com esse pensamento, Sheldon e King (2001) sugerem que os psicólogos adotem essa perspectiva por considerar as motivações humanas nos diversos momentos e situações de vida. Consequentemente, a resiliência é um construto convergente e alvo de interesse de pesquisas em psicologia nos últimos trinta anos, sendo, entretanto, anterior à própria psicologia positiva, que recentemente a incorporou como temática de interface (Masten \& Garmezy, 1985; Rutter, 1993, 1996).

Verifica-se na literatura da área, diversos trabalhos embasados na psicologia positiva e promoção do bem-estar para o entendimento de temáticas relacionadas à área da saúde mental (p. ex., Carlton et al., 2006; Passareli \& Silva, 2007; Poletto \& Koller, 2006). Diante dessa 
constatação, o fortalecimento e a construção das forças pessoais pode propiciar o que Keyes e Haidt (2003) e Seligman (2011) chamam de florescimento (flourishing) das potencialidades das pessoas, comunidades e instituições, ou seja, o desenvolvimento pleno, saudável e positivo dos aspectos biopsicossociais dos indivíduos.

Como resultado, Seligman (2011) enfatiza que os conceitos da psicologia positiva, como competências do bem-estar (ter mais emoções positivas, mais sentido, engajamento e relacionamentos mais satisfatórios), por exemplo, devem ser ensinados na escola, uma vez que, melhoram a aprendizagem, produzem maior atenção e pensamento criativo, e previnem depressão. $\mathrm{O}$ que é reforçado pelas considerações de Csikszentmihalyi (2009), é o fato de que esse movimento deve não apenas se preocupar com a forma como as pessoas sentem uma emoção positiva, mas de refletir sobre a maneira como elas vivem e fazem suas escolhas.

A partir desse contexto, alguns fenômenos vêm sendo apontados como sistemas de construção saudável ao longo do desenvolvimento, em especial, as competências do bem-estar referidas (Seligman, 2011), e a resiliência (Rutter, 1996), que na literatura está vinculada aos conceitos de fatores de risco e de fatores de proteção.

Desse modo, segundo Poletto e Koller (2006), os fatores de risco consistem nas condições que estão aliadas à elevada possibilidade de ocorrência de resultados negativos, que podem comprometer a saúde, o bem-estar ou desempenho social do adolescente. Dentre os fatores de risco tem-se: eventos estressantes de vida, carência de apoio social e afetivo, déficits em habilidades sociais, cognitivas e de manejo das emoções (Masten \& Garmezy, 1985). Já os fatores de proteção, segundo os primeiros autores, referem-se às influências que modificam ou melhoram respostas pessoais a determinados riscos de desadaptação, o que produz efeitos positivos na saúde mental. Masten e Garmezy (1985) identificaram três classes de fatores protetivos: os atributos disposicionais das pessoas como o bem-estar, orientação social positiva, autonomia; os laços afetivos no sistema familiar e/ou em outros contextos que ofereçam suporte emocional; os sistemas de rede de apoio social, seja na escola, no trabalho, no serviço de saúde, propiciando o desenvolvimento positivo.

Tendo como base os pressupostos apresentados, destaca-se que a aplicação desses princípios pela psicologia positiva reforça a promoção de competências sociais, emocionais e cognitivas proporcionando a maximização das potencialidades dos jovens e a minimização dos fatores de risco à saúde (J. V. Lerner et al., 2009). Portanto, reconhecendo que é neste período do ciclo vital em que pode ser alta a ocorrência de dificuldades de aprendizagem, transtornos afetivos e de conduta, baixos níveis de motivação e realização, além de envolvimento com álcool e drogas ilícitas é importante promover o desenvolvimento na adolescência de maneira integral, com o foco direcionado aos modelos de desenvolvimento enfatizando como os adolescentes crescem, aprendem e mudam (Catalano et al., 2004; Damon, 2004).

Visando compreender essa nova visão, no decorrer desse estudo dialogou-se com referências teórico-conceituais que têm norteado questões de intervenções no âmbito da promoção do desenvolvimento positivo do jovem. Deste modo, é relevante apontar que as intervenções proativas também são fundamentadas em estudos que vêm corroborando a sua efetividade (Catalano et al., 2004; Mangrulkar, Whitman, \& Posner, 2001; Seligman, 2011; Vicary et al., 2006), legitimando, assim, a sua aplicabilidade em diferentes contextos, a partir de um compromisso científico orientado a interligar a teoria e a prática.

Contudo, como pode ser verificado por meio dos resultados de revisões da literatura especializada em prevenção primária em saúde (Durlak \& Wells, 1997; Kulic, Horne, \& Dagley, 2004; Murta, 2007) na infância e adolescência, há uma aplicação considerável da abordagem cognitivo-comportamental (TCC) nos programas preventivos.

Por conseguinte, constata-se que essa abordagem se aproxima da psicologia positiva quanto às técnicas aplicadas, entretanto, ambas apresen- 
tam pressupostos teóricos e visões de ser humano bastante peculiares. Por exemplo, a TCC tem demonstrado evidências empíricas sobre sua efetividade no tratamento de psicopatologias, com mais de 350 estudos sobre o desfecho positivo de sua aplicação (Bieling, McCabe, \& Antony, 2008). Portanto é evidente o seu auxílio na amenização de sintomas e no alívio do sofrimento imediato.

Apesar da comprovada eficácia da TCC é importante lembrar que a ausência de doenças não equivale à presença de saúde e bem-estar, de acordo com os princípios da psicologia positiva (Keyes \& Haidt, 2003). Desse modo Seligman (2011) admite que a produção e ampliação das emoções positivas resultam em recursos psicológicos duradouros dos quais as pessoas podem vir a usufruir mais tarde na vida. Assim, mesmo que a TCC ofereça uma ampla gama de estudos com evidências de efetividade, ainda é possível potencializar os efeitos de uma intervenção a partir do desenvolvimento de aspectos positivos do indivíduo.

\section{Progressos das Ações Interventivas com Ideário Positivo}

De acordo com Gable e Haidt (2005) o estudo das qualidades positivas encontra-se em desenvolvimento, podendo, assim, promover oportunidades de um crescimento rápido no conhecimento da Psicologia, bem como a utilização de novas estratégias no tratamento das desordens mentais. Ao longo da história, processou-se o desenvolvimento da prevenção primária na Psicologia culminando no acúmulo de conhecimentos sobre a origem dos comportamentos não adaptativos e prejudiciais à saúde, com enfoque clínico e remediativo de intervenção (Albee, 2003; Lacerda \& Guzzo, 2005).

Nesse sentido, o conhecimento acerca dos fatores de risco individuais proliferou amplamente entre os pesquisadores norte-americanos dando origem a programas de treinamento específicos (Chalk, Phillips, \& National Research Council [NRC], Institute of Medicine [IOM], 1996). Posteriormente, com a comprovação das influências sociais e familiares na prevenção de problemas infanto-juvenis ocorreu o surgimento de atividades voltadas à melhoria da saúde em comunidades estadunidenses.

A partir destas propostas houve uma mudança no foco dos estudos na área de prevenção. No lugar da investigação de problemas, como a delinquência juvenil e o abuso de drogas, passou-se a se dedicar à pesquisa de fatores sociais envolvidos em interações positivas de crianças e adolescentes com suas famílias, pares e escola (Catalano et al., 2004). Com esses avanços, confirma Seligman (2011), as intervenções passaram a se direcionar para os fatores protetivos apontando a promoção da saúde, reforçada pelo movimento da psicologia positiva, como uma nova estratégia a ser adotada pelos programas interventivos.

A opção por uma atuação promotora de saúde levou ao surgimento de programas direcionados à melhoria da qualidade de vida e bem-estar por meio da implementação de intervenções também em outros países, como citado por Seligman (2011), por exemplo, no Reino Unido, Austrália, China e Portugal. Segundo o autor, em cada país os programas foram desenvolvidos respeitando as diversidades regionais, tendo em comum à proposta de fortalecimento de competências associadas à saúde entre jovens numa ótica proativa, em direção à redução dos comportamentos de risco.

Nessa perspectiva, Catalano et al. (2004) foram os pioneiros em realizar uma revisão sistemática dos programas de desenvolvimento positivo americanos identificando 25 programas de sucesso. Os autores basearam-se em critérios rígidos para a seleção destes programas visando à construção de uma definição operacional do termo Desenvolvimento Positivo. A partir disso, encontraram um total de 15 construtos representativos dessa operacionalização e, sendo assim, compreenderam que se um ou mais dos construtos estiverem presentes, o foco recairá sobre o Desenvolvimento Positivo, a saber: promoção de vínculos seguros, competência social, emocional, cognitiva, comportamental, moral, auto-eficácia, normas pró-sociais, resiliência, autodeterminação, espiritualidade, crença no futuro, identidade clara e positiva, oportunidades para o 
envolvimento pró-social e o reconhecimento do comportamento positivo.

No tocante aos resultados da implementação dos 25 programas desta revisão observou-se, segundo Catalano et al. (2004), melhorias significativas nas habilidades interpessoais, no autocontrole, na solução de problemas, nas competências cognitivas e na auto-eficácia, assim como redução de diversos problemas de comportamento (p. ex., violência, mau comportamento escolar, uso de álcool e drogas). Deste modo, verifica-se que as intervenções positivas que abordam construtos do desenvolvimento positivo estão, concretamente, fazendo a diferença em estudos bem avaliados.

As evidências da efetividade da implementação destes programas são utilizadas como fundamento não somente por diminuírem a incidência, prevalência e recorrência de problemas comportamentais e mentais, mas, sobretudo, como afirma Seligman (2011), por promoverem o bem-estar por meio de instrumentos validados, métodos testados e comprovados de avaliação, experimentação, pesquisas longitudinais e estudos com distribuição aleatória, e grupo controle, para avaliar quais intervenções efetivamente funcionam e quais são ineficazes.

Uma meta-análise efetuada por Durlak, Weissberg e Pachan (2010) avaliou 69 programas estadunidenses pós-escola que objetivaram melhorar a competência pessoal e social de jovens entre 5 e 18 anos. Os resultados indicaram que, comparados aos grupos controles, os participantes dos grupos experimentais evidenciaram aumento significativo na auto-percepção, no vínculo com a escola, nos comportamentos sociais, nos níveis de desempenho acadêmico, bem como reduções significativas nos comportamentos problemáticos.

Outras intervenções positivas direcionadas à saúde entre jovens foram desenvolvidas, tanto no contexto nacional como internacional, por diferentes pesquisadores (Botvin \& Griffin, 2004; Castellanos, 2001; Gorayeb et al., 2003; Mangrulkar et al., 2001; Minto, 2005), como é o caso do programa de Ensino de Habilidades de Vida, preconizado pela World Health Organization (WHO, 1997). O programa é composto por 10 competências que influenciam o comportamento humano, sendo consideradas de grande relevância nos processos de intervenção. As 10 competências são: tomada de decisão, resolução de problemas, pensamento crítico e criativo, comunicação eficaz, relacionamento interpessoal, autoconhecimento, empatia, manejo de emoções e de estresse (WHO, 1997).

Segundo Castellanos (2001), este programa permite que os adolescentes tenham a oportunidade de adquirir novos conhecimentos, além de pretender influenciar diretamente na formação de seus valores e atitudes. Inicialmente, os programas de habilidades de vida foram implementados com o objetivo de diminuir o comportamento do uso de tabaco (Botvin \& Eng, 1982; Botvin, Eng, \& Williams, 1980), porém obtiveram avanços para a prevenção ao consumo de álcool, outras drogas e problemas comportamentais diversos, a partir do princípio da promoção de competências psicossociais, com vistas à promoção de saúde na adolescência.

Para exemplificar esse progresso, um trabalho de mais de 20 anos conduzido por Botvin e colaboradores (Botvin \& Griffin, 2004), com adolescentes em escolas estadunidenses e baseado no Life Skills Training (LST) para a prevenção ao consumo de substâncias psicoativas, abarcou os três eixos: o desenvolvimento de habilidades de controle pessoal, o incremento de competências sociais, e também a ampliação do conhecimento dos jovens no que concerne às drogas e das competências de resistência a elas. O programa foi realizado no período de três anos, tendo início com os alunos da $7^{\mathrm{a}}$ série do ensino fundamental. Durante o primeiro ano foram realizadas 15 sessões, com 45 minutos de duração. Nos dois anos seguintes, foram promovidas 10 sessões de reforço com os alunos da $8^{\mathrm{a}}$ série e, no último ano, foram feitas cinco sessões com os jovens do $1^{\circ}$ ano do ensino médio. Foram desenvolvidos materiais curriculares para a implementação do programa - manual do professor e um guia do estudante. $\mathrm{O}$ método utilizado apoiou-se em técnicas cognitivo-comportamentais, ensino de habilidades sociais e discussão em grupo. Segundo os autores, as habilidades revelaram-se como protetivas por reduzir as expectativas po- 
sitivas do jovem frente às drogas e auxiliar no aumento da comunicação.

Com o objetivo de avaliar a efetividade do trabalho supracitado, Vicary et al. (2006) realizaram um estudo prospectivo denominado ADAPT (adoção de Treinamento de Prevenção do Abuso de Drogas) para comparar dois métodos de aplicação do LST. Os autores avaliaram os resultados do LST em jovens escolares. Os participantes foram distribuídos dentro de três condições: (a) LST padrão (108 mulheres, 126 homens) realizado por três anos, conforme mencionado anteriormente; (b) I-LST (128 mulheres, 169 homens) sem conter sessões previamente definidas; (c) condição controle (98 mulheres, 103 homens), sem tratamento. As avaliações foram realizadas com questionários, aplicados em quatro tempos diferentes (T1 - pré-intervenção/ T2 - final da $7^{\text {a }}$ série /T3 - final da $8^{\text {a }}$ série /T4 - final do $1^{\circ}$ ano), englobando o comportamento dos alunos quanto ao uso de substâncias; as crenças com relação às mesmas; e habilidades de comunicação, tomada de decisão, resistência e recusa. Como resultado, o LST padrão foi mais eficaz nas avaliações iniciais realizadas no T2 quanto ao uso de substâncias; e as habilidades de tomada de decisão, comunicação e enfrentamento para as mulheres. Já o I-LST apresentou efeito positivo no $\mathrm{T} 2$ apenas quanto ao conhecimento em relação às drogas, para as mulheres.

Nessa direção, tendo em vista os resultados dos estudos supracitados evidenciando aspectos favoráveis à efetividade de intervenções com vistas à promoção de competências do bem-estar entre os jovens, verifica-se, simultaneamente, a necessidade de maior divulgação e a viabilidade de tais intervenções no cenário nacional. Conforme apontado por Seligman (2011), cada uma destas competências pode ser utilizada em diversas áreas do funcionamento psicossocial dos jovens, ampliando ações positivas no futuro.

\section{Contextualizando as Pesquisas Nacionais}

No presente artigo empreendeu-se uma síntese de aspectos conceituais e empíricos que embasam programas interventivos na adolescência, considerando ser esta uma área que está incluída no campo da prevenção e promoção da saúde mental. A tarefa fundamental dos programas com enfoque proativo é fomentar a melhoria do bem-estar e a qualidade de vida dos adolescentes, e consequentemente o de sua família, frente a situações adversas, considerando a saúde um conceito multidimensional que abarca os aspectos físicos, psicológicos e sociais interligados ao desenvolvimento positivo.

De modo geral, são escassas as investigações que priorizam as medidas da psicologia positiva no contexto brasileiro. Portanto, Scorsolini-Comin e Santos (2010) afirmam que as pesquisas no campo ainda são incipientes. Por conseguinte, o acúmulo de achados promissores dos estudos referidos, e daqueles que são retratados em publicações encontradas no Brasil, vêm apontando ser este um campo fértil para a produção de saber científico de impacto vinculado à construção do bem-estar e das competências positivas (Albuquerque \& Tróccoli, 2004; Morais \& Koller, 2004; Paludo \& Koller, 2007; Passareli \& Silva, 2007), o que torna pertinente sua investigação em diferentes culturas jovens.

Apesar de serem poucas as intervenções realizadas no Brasil, destacadamente alinhadas à psicologia positiva, observa-se um crescimento nesta área por meio da realização de estudos no campo da saúde (Strelhow, Bueno, \& Câmara, 2010), bem como da validação e avaliação dos indicadores do bem-estar - afeto positivo, afeto negativo e satisfação com a vida (Albuquerque \& Tróccoli, 2004; Arteche \& Bandeira, 2003; Noronha \& Mansão, 2012; Segabinazi, Giacomoni, Dias, Teixeira, \& Moraes, 2010; Segabinazi et al., 2012). Pois, conforme Seligman e Csikszenmihalyi (2000), a negligência em relação ao estudo dos aspectos positivos e virtuosos dos indivíduos pela ciência psicológica é resultante da expansão das investigações sobre os aspectos negativos em detrimento dos positivos, devido ao enfoque clínico e remediativo das intervenções.

Partindo desses pressupostos, Arteche e Bandeira (2003) analisaram a existência de diferenças em relação aos níveis de bem-estar em 193 adolescentes de escolas públicas, tendo 112 
meninos (58\%) e 81 meninas ( $42 \%)$, com idades entre 14 e 17 anos. Os participantes foram divididos em três grupos: um grupo de trabalhadores em regime educativo $(n=58)$, trabalhadores em regime regular $(n=58)$ e o terceiro grupo de não-trabalhadores $(n=77)$. Utilizou-se, dentre outros instrumentos, a Escala Multidimensional de Satisfação de Vida e as Escalas de Afeto Positivo e Afeto Negativo (PANAS). Os resultados evidenciaram que o trabalho para os adolescentes em regime educativo foi positivo, os quais indicaram mais satisfação em suas vidas. No que diz respeito às escalas PANAS, não foram encontradas correlações significativas $(r=0,12)$ entre os componentes afetivos. Porém, em relação aos escores médios de afeto positivo verificou-se serem superiores ao de afeto negativo.

De modo em especial, os adolescentes precisam ser orientados para a transição da educação para o mundo do trabalho, com o propósito de obterem clareza de suas habilidades e metas profissionais. Nesse sentido, Noronha e Mansão (2012) realizaram um estudo exploratório entre interesses profissionais e afetos positivos e negativos de jovens. Participaram 529 estudantes do ensino médio, com idades entre 14 a 27 anos. Quanto ao sexo, 223 (42,2\%) eram homens e 306 (57,8\%), mulheres. Aplicou-se a EAZ - Escala de Afetos de Zanon e a EAP - Escala de Aconselhamento Profissional. Os participantes apresentaram maiores médias para afetos positivos, o que indica julgamento mais positivo da vida, de modo geral. A comparação entre os instrumentos revelou que das 14 correlações encontradas, oito delas apresentaram coeficientes positivos e significativos, mas de baixa magnitude.

Com isso, mesmo não sendo de intervenção os dois estudos mencionados, de acordo com o que foi explorado inicialmente por Botvin e Griffin (2004), Catalano et al. (2004), Durlak et al. (2010), e vem sendo investigado também por pesquisadores brasileiros (Gorayeb et al., 2003; Minto, 2005), o incremento do bem-estar e das competências positivas na adolescência podem favorecer o desenvolvimento positivo do jovem por meio de intervenções efetivas.

Para tanto, verifica-se também no Brasil algumas tentativas de desenvolvimento de in- tervenções alinhadas aos princípios da psicologia positiva com base no ensino de habilidades de vida (Minto, 2005) e de habilidades sociais (Silva \& Murta, 2009). Enquanto o termo habilidades de vida aplica-se, como já foi dito, a um amplo conjunto de comportamentos envolvidos no enfrentamento de demandas da vida cotidiana (Gorayeb et al., 2003); o termo habilidades sociais, por sua vez, compõe uma categoria conceitual mais delimitada. Refere-se ao conjunto de classes de comportamentos sociais do repertório do indivíduo, que contribuem para a competência social, viabilizando um relacionamento interpessoal saudável e produtivo (Del Prette \& Del Prette, 2011).

Tal empreendimento nacional pode ser exemplificado pela intervenção conduzida por Minto (2005) avaliando os efeitos do ensino de habilidades de vida em 45 adolescentes, com idades entre 14 e 17 anos, maioria do sexo masculino, vinculados a duas instituições profissionalizantes em Ribeirão Preto/SP. Formaram-se quatro grupos por instituição, com média de seis participantes em cada. Realizou-se 16 encontros, avaliados a partir de um questionário sobre comportamentos de risco e a escala de Locus de Controle (percepção de controle sobre eventos de vida), aplicados pré e pós-intervenção, e efetuando entrevista final com os participantes. A metodologia foi interativa, com dramatizações e discussões. Os resultados em ambas as instituições não demonstraram diferenças significativas, contudo, a partir dos relatos dos participantes observou-se que as habilidades de vida (lidar com o stress e os sentimentos) foram aprimoradas. Na conclusão da autora, a aprendizagem sobre como utilizar essas habilidades pode facilitar respostas mais ajustadas para os jovens no futuro.

Outra intervenção grupal conduzida por Silva e Murta (2009) focalizou as habilidades sociais. Participaram 12 adolescentes com idade entre 11 e 14 anos, maioria do sexo masculino, vinculados ao Programa de Atenção Integral à Família (PAIF) de uma cidade do interior de Goiás. A intervenção foi feita em 11 sessões grupais visando avaliar as atitudes de (in)satisfação dos participantes frente ao programa e o alcance 
de metas intermediárias previstas para o mesmo, consideradas indicadores de comprometimento com o grupo. Os resultados revelaram satisfação frente à intervenção, principalmente no que se refere à liberdade para se expressar e aos efeitos positivos da intervenção. Os indicadores de comprometimento com o grupo de maior frequência foram relatar sentimentos, relatar problemas, explicar a causa do próprio comportamento e oferecer apoio ao colega.

Portanto, de acordo com as premissas da psicologia positiva, os profissionais que atuam com adolescentes ao possibilitarem a construção de formas satisfatórias de resolução das tensões cotidianas, podem favorecer, como afirma Ungar (2008), o fortalecimento dos adolescentes e a sensação de bem-estar. Tais aspectos são fundamentais para o processo de resiliência, para o potencial de desenvolvimento do poder pessoal, senso de autonomia, capacidade para transformação pessoal e social favorável, além do estabelecimento de vínculos significativos com adultos que garantam a confiança em pessoas e instituições.

Senna e Dessen (2012) ressaltam as potencialidades da visão positiva do desenvolvimento na juventude como tentativa de romper o viés patologizante (negativo) e despertar a comunidade acadêmica brasileira para acompanhar os avanços nessa área. Portanto, temáticas como as habilidades psicossociais, as competências do bem-estar e a resiliência destacam-se nos atuais estudos científicos da psicologia.

\section{Considerações Finais}

Ressalta-se a relevância de se promoverem as habilidades psicossociais e competências de bem-estar, conforme destacado pelos autores, em adolescentes de diferentes contextos e nacionalidades (Arteche \& Bandeira, 2003; Catalano et al., 2004; Durlak et al., 2010; Minto, 2005; Seligman, 2011). Os resultados dos estudos mencionados evidenciaram a diminuição de diversos problemas comportamentais e emocionais na adolescência. Verificou-se, contudo, um incremento notório das habilidades cognitivas, emocionais e sociais, o que reflete a mudança de foco dos estudos em relação aos aspectos negativos para os positivos do desenvolvimento humano.

Há que se implementar intervenções na adolescência, como preconiza a Psicologia Positiva (Seligman, 2011), não somente para conduzir a resolução de transtornos, mas, sobretudo, favorecer a criação de oportunidades para o desenvolvimento das potencialidades de maneira efetiva. Sob essa perspectiva, é possível permitir que os jovens consigam superar o déficit em suas competências, tornando-se inclusive os protagonistas de sua própria vida ao fortalecerem sua capacidade para crescerem e se desenvolverem de modo saudável.

Contudo, torna-se necessário considerar que apesar desse avanço nos programas interventivos pode-se verificar uma tendência da aplicação de abordagens direcionadas mais para o alívio de sintomas imediatos, que para a promoção do bem-estar e da qualidade de vida. Portanto, recomenda-se que sejam realizadas pesquisas sobre a temática do desenvolvimento positivo enquanto base teórico-metodológica para programas de intervenção na adolescência. Para tanto, torna-se relevante englobar a saúde de modo integral, adotando o fortalecimento das competências para a vida, e investindo sobre o bem-estar e a resiliência em total sincronia com a perspectiva da psicologia positiva.

Por fim, nota-se que o movimento da psicologia positiva traz à tona importantes implicações teóricas, empíricas e práticas para o investimento em prevenção e promoção da saúde mental dos jovens, na medida em que, dentre outros aspectos de relevância, pode viabilizar a contenção de despesas pelo sistema público brasileiro no que tange à saúde e educação. $\mathrm{O}$ que denota, diante da escassez de pesquisas com intervenção alinhadas às premissas da psicologia positiva no Brasil, a pertinência do seu desenvolvimento de modo efetivo nos programas interventivos nacionais, tal como ocorre no cenário internacional. E desse modo, ao priorizar as ações positivas, enfatizando e promovendo forças e virtudes próprias do ser adolescente pode ser possível construir oportunidades para que os mesmos aperfeiçoem as suas potencialidades, praticando e descobrindo novas habilidades. 


\section{Referências}

Albee, G. W. (2003). Confrontations and change. In D. K. Freedheim \& I. B. Weiner (Eds.), Handbook of psychology: Vol. 1. History of psychology (pp. 486-488). Hoboken, NJ: John Wiley \& Sons.

Albuquerque, A. S., \& Tróccoli, B. T. (2004). Desenvolvimento de uma escala de bem-estar subjetivo. Psicologia: Teoria e Pesquisa, 20(2), 153164. doi:10.1590/S0102-37722004000200008

Allport, G. W. (1958). Becoming: Basic considerations for a psychology of personality. Oxford, UK: Verlag.

Amparo, D. M., Galvão, A., Alves, P., Cardenas, C., \& Koller, S. (2008). A escola e as perspectivas educacionais de jovens em situação de risco. Psicologia Escolar e Educacional, 12, 69-88. doi:10.1590/S1413-85572008000100006

Arteche, A. X., \& Bandeira, D. R. (2003). Bem-estar subjetivo: Um estudo com adolescentes trabalhadores. Psico-USF, 8(2), 193-201.

Bandura, A. J. (1977). Social learning theory. Englewood Cliffs, NJ: Prentice Hall.

Benson, P. L., Scales, P. C., Hamilton, S. F., Sesma, A., Jr., Hong, K. L., \& Roehlkepartain, E. C. (2006). Positive youth development so far core hypotheses and their implications for policy and practice. Search Institute Insights \& Evidence, 3(1), 1-13.

Bieling, P. J., McCabe, R. E., \& Antony, M. M. (2008). Terapia cognitivo-comportamental em grupos. Porto Alegre, RS: Artmed.

Botvin, G. J., \& Eng, A. (1982). The efficacy of a multicomponent approach to the prevention of cigarette smoking. Preventive Medicine, 11, 199-211. doi:10.1016/0091-7435(82)90018-4

Botvin, G. J., Eng, A., \& Williams, C. L. (1980). Preventing the onset of cigarette smoking through life skills training. Journal of Preventive Medicine, 9(1), 135-143. doi:10.1016/00917435(80)90064-X

Botvin, G. J., \& Griffin, K. W. (2004). Life skills training: Empirical findings and future directions. The Journal of Primary Prevention, 25(2), 211-232. doi:10.1023/B:JOPP.0000042391.58573.5b

Bowlby, J. (1982). Attachment. Attachment and loss ( $2^{\text {nd }}$ ed.). New York: Basic Books.
Brasil, K., Alves, P., Amparo, D. M., \& Frajorge, K. (2006). Fatores de risco na adolescência: Discutindo dados do DF. Paidéia (Ribeirão Preto), 16(35), 377-384. doi:10.1590/S0103$-863 \times 2006000300008$

Bronfenbrenner, U. (1996). A ecologia do desenvolvimento humano: Experimentos naturais $e$ planejados (M. A. Veríssimo, Trad.). Porto Alegre, RS: Artes Médicas. (Original publicado em 1979)

Cardozo, G., Dubini, P., Fantino, I., \& Ardiles, R. (2011). Habilidades para la vida en adolescentes: diferencias de género, correlaciones entre habilidades y variables predictoras de la empatía. Psicología desde el Caribe, 28, 107-132.

Carlton, B. S., Goebert, D. A., Miyamoto, R. H., Andrade, N. N., Hishinuma, E. S., Makini, G. K. Jr., ...Nishimura, S. T. (2006). Resilience, family adversity and well-being among Hawaiian and non-Hawaiian adolescents. International Journal of Social Psychiatry, 52(4), 291-308. doi:10.1177/0020764006065136

Castellanos, M. L. (2001). Habilidades para la vida. Uma propuesta educativa para la promoción del desarolllo humano y la prevención de problemas psicossociales. Bogotá, Colômbia: Fe y Alegría.

Catalano, R. F., Berglund, M. L., Ryan, J. A. M., Lonczak, H. S., \& Hawkins, J. D. (2004). Positive youth development in the United States: Research findings on evaluations of positive youth development programs. The Annals of the American Academy of Political and Social Science, 591(1), 98-124. doi:10.1177/0002716203260102

Chalk, R., Phillips, D. A., \& National Research Council, Institute of Medicine. (Eds.). (1996). Youth development and neighborhood influences: Challenges and opportunities. Summary of a workshop (Report by the Committee on Youth Development, Board on Children, Youth, and Families, Commission on Behavioral and Social Sciences and Education). Washington, DC: National Academy Press.

Cowan, E. (2000). Psychological wellness: Some hopes for the future. In D. Cicchetti, J. Rappaport, I. Sandler, \& R. P. Weissberg (Eds.), The promotion of wellness in adolescents (pp. 477503). Washington, DC: Child Welfare League of America Press. 
Csikszentmihalyi, M. (2009). The promise of positive psychology. Psychological Topics, 18(2), 203211.

Damon, W. (2004). What is positive youth development? The Annals of the American Academy of Political and Social Science, 591(1), 13-24.

De Antoni, C., \& Koller, S. H. (2000). A visão de família entre as adolescentes que sofreram violência intrafamiliar. Estudos de Psicologia (Natal), 5(2), 347-381.

Del Prette, Z. A. P., \& Del Prette, A. (2011). Psicologia das habilidades sociais na infância: Teoria e prática (5. ed.). Petrópolis, RJ: Vozes.

Durlak, J. A., Weissberg, R. P., \& Pachan, M. (2010). A meta-analysis of after-school programs that seek to promote personal and social skills in children and adolescents. American Journal of Community Psychology, 45(3-4), 294-309. doi:10.1007/s10464-010-9300-6

Durlak, J. A., \& Wells, A. M. (1997). Primary prevention mental health programs for children and adolescents: A meta-analytic review. American Journal of Community Psychology, 25(2), 115152. doi:10.1023/A:1024654026646

Erikson, E. (1976). Identidade, juventude e crise (2. ed.). Rio de Janeiro, RJ: Zahar. (Original publicado em 1968)

Gable, S. L., \& Haidt, J. (2005). What (and why) is positive psychology? Review of General Psychology, 9(2), 103-110. doi:10.1037/10892680.9.2.103

Goosens, L. (2006). Adolescent development: Putting Europe on the map. In S. Jackson \& L. Goosens (Eds.), Handbook of adolescent development (pp. 1-10). New York: Psychology Press.

Gorayeb, R., Netto, J. R. C., \& Bugliani, M. A. P. (2003). Promoção de saúde na adolescência: Experiência com programas de ensino de habilidades de vida. In Z. A. Trindade \& A. N. Andrade (Eds.), Psicologia e saúde: Um campo em construção (pp. 89-100). São Paulo, SP: Casa do Psicólogo.

Hall, G. S. (1904). Adolescence: Its psychology and its relations to physiology, anthropology, sociology, sex, crime, religion, and education (Vol. 2). New York: Appleton.

Heilborn, M. L., Salem, T., Rohden, F., Brandão, E., Knauth, D., Víctora. C, ...Bozon, M. (2002).
Aproximações socioantropológicas sobre a gravidez na adolescência. Horizontes Antropológi$\cos , 8(17), 13-45$.

Inhelder, B., \& Piaget, J. (1976). Da lógica da criança a lógica da adolescente: Ensaio sobre a construção das estruturas operatórias formais (D. M. Leite, Trad.). São Paulo, SP: Pioneira. (Original publicado em 1958)

James, W. (1902). The varieties of religious experience: A study in human nature. New York: Longman.

Keyes, C. L. M., \& Haidt, J. (Eds.). (2003). Flourishing: Positive psychology and a life well lived. Washington, DC: American Psychological Association.

Kulic, K. R., Horne, A. M., \& Dagley, J. C. (2004). A comprehensive review of prevention groups for children and adolescents. Group Dynamics: Theory, Research and Practice, 8, 139-151.

Lacerda, F., Jr., \& Guzzo, R. S. L. (2005). Prevenção primária: Análise de um movimento e possibilidades para o Brasil. Interação em Psicologia, 9(2), 239-249.

Lerner, J. V., Phelps, E., Forman, Y., \& Bowers, E. P. (2009). Positive youth development. In R. M. Lerner \& L. Steinberg (Eds.), Handbook of adolescent psychology ( $3^{\text {rd }}$ ed., pp. 524-558). Hoboken, NJ: Wiley.

Lerner, R. M., \& Galambos, N. L. (1998). Adolescent development: Challenges and opportunities for research, programs, and policies. Annual Review of Psychology, 49, 413-446.

Lerner, R. M., \& Steinberg, L. (2009). The scientific study of adolescent development: Historical and contemporary perspectives. In R. M. Lerner \& L. Steinberg (Eds.), Handbook of adolescent psychology ( $3^{\text {rd }}$ ed., pp. 3-14). Hoboken, NJ: Wiley.

Mangrulkar, L., Whitman, C. V., \& Posner, M. (2001). Enfoque de habilidades para la vida para un desarrollo saludable de niños y adolescentes. Washington, DC: Organización Panamericana de la Salud.

Marques, A. C., \& Cruz, M. S. (2000). O adolescente e o uso de drogas. Revista Brasileira de Psiquiatria, 22(2), 32-36.

Maslow, A. H. (1968). Toward a psychology of being. New York: Van Nostrand. 
Masten, A. S., \& Garmezy, N. (1985). Risk, vulnerability and protective factors in developmental psychopathology. In B. B. Lahey \& A. E. Kazdin (Eds.), Advances in child clinical psychology (Vol. 8, pp. 1-52). New York: Plenum Press.

Mead, M. (1979). Coming of age in Samoa: A psychological study of primitive youth for Western civilization. New York: Norton. (Original publicado em 1928)

Minto, E. C. (2005). Ensino de habilidades de vida para adolescentes vinculados a instituições profissionalizantes, no município de Ribeirão Preto/SP (Dissertação de mestrado, Universidade de São Paulo, Ribeirão Preto, SP, Brasil).

Moore, K. A., \& Keyes, C. L. M. (2003). A brief history of the study of well-being in children and adults. In M. H. Bornstein, L. Davidson, C. L. M. Keyes, \& K. A. Moore (Eds.), Well-being: Positive development across the life course (pp. 1-11). Mahwah, NJ: Lawrence Erlbaum.

Morais, N. A., \& Koller, S. H. (2004). Abordagem ecológica do desenvolvimento humano, psicologia positiva e resiliência: A ênfase na saúde. In S. H. Koller (Ed.), Ecologia do desenvolvimento humano: Pesquisa e intervenção no Brasil. (pp. 91-107). São Paulo, SP: Casa do Psicólogo.

Murta, S. G. (2007). Programas de prevenção a problemas emocionais e comportamentais em crianças e adolescentes: Lições de três décadas de pesquisa. Psicologia: Reflexão e Crítica, 20(1), $1-8$.

Noronha, A. P. P., \& Mansão, C. S. M. (2012). Interesses profissionais e afetos positivos e negativos: Estudo exploratório com estudantes de ensino médio. Psico-USF, 17(2), 323-331. doi:10.1590/S1413-82712012000200016

Paludo, S., \& Koller, S. H. (2007). Psicologia positiva: Uma nova abordagem para antigas questões. Paidéia (Ribeirão Preto), 17(36), 9-20. doi:10.1590/S0103-863X2007000100002

Passareli, P. M., \& Silva, J. A. (2007). Psicologia positiva e o estudo do bem-estar subjetivo. Estudos de Psicologia (Campinas), 24(4), 513-517. doi:10.1590/S0103-166X2007000400010

Pesce, R., Assis, S., Santos, N., \& Oliveira, N. (2005). Risco e proteção: Em busca de um equilíbrio promotor de resiliência. Psicologia: Teoria e Pesquisa, 20(2), 135-143.
Poletto, M., \& Koller, S. H. (2006). Resiliência: Uma perspectiva conceitual e histórica. In D. D. Dell'Aglio, S. H. Koller, \& M. A. M. Yunes (Eds.), Resiliência e psicologia positiva: Interfaces do risco à proteção (pp. 19-44). São Paulo, SP: Casa do Psicólogo.

Rutter, M. (1993). Resilience: Some conceptual considerations. Journal of Adolescent Health, 14(8), 626-631. doi:10.1016/1054-139X(93)90196-V

Rutter, M. (1996). Psychosocial resilience and protective mechanisms. In J. Rolf, A. S. Masten, D. Cicchetti, K. H. Nuechterlein, \& S. Weintraub (Eds.), Risk and protective factors en the development of psychology (pp. 181-214). New York: Cambridge University.

Santrock, J. W. (2014). Adolescência (14. ed.). Porto Alegre, RS: AMGH.

Scorsolini-Comin, F., \& Santos, M. A. (2010). Psicologia positiva e os instrumentos de avaliação no contexto brasileiro. Psicologia: Reflexão $e$ Crítica, 23(3), 440-448. doi:10.1590/S010279722010000300004

Segabinazi, J. D., Giacomoni, C. H., Dias, A. C. G., Teixeira, M. A. P., \& Moraes, D. A. O. (2010). Desenvolvimento e validação preliminar de uma escala multidimensional de satisfação de vida para adolescentes. Psicologia: Teoria $e$ Pesquisa, 26(4), 653-659. doi:10.1590/S010237722010000400009

Segabinazi, J. D., Zortea, M., Zanon, C., Bandeira, D. R., Giacomoni, C. H., \& Hutz, C. S. (2012). Escala de afetos positivos e negativos para adolescentes: Adaptação, normatização e evidências de validade. Avaliação Psicológica, 11(1), 1-12.

Seligman, M. E. P. (2011). Florescer: Uma nova compreensão sobre a natureza da felicidade $e$ do bem-estar. Rio de Janeiro, RJ: Objetiva.

Seligman, M. E. P., \& Csikszentmihalyi, M. (2000). Positive psychology: An introduction. American Psychologist Association, 55(1), 5-14. doi:10.1037/0003-066X.55.1.5

Senna, S. R. C. M., \& Dessen, M. A. (2012). Contribuições das teorias do desenvolvimento humano para a concepção contemporânea da adolescência. Psicologia: Teoria e Pesquisa, 28(1), 101108. doi:10.1590/S0102-37722012000100013

Sheldon, K. M., \& King, L. (2001). Why positive psychology is necessary. American Psychologist, 56(3), 216-217. 
Silva, M. P., \& Murta, S. G. (2009). Treinamento de habilidades sociais para adolescentes: Uma experiência no programa de atenção integral à família (PAIF). Psicologia: Reflexão e Crítica, 22(1), 136-143. doi:10.1590/S010279722009000100018

Strelhow, M. R. W., Bueno, C. O., \& Câmara, S. G. (2010). Percepção de saúde e satisfação com a vida em adolescentes: Diferença entre os sexos. Revista Psicologia e Saúde, 2(2), 42-49.

Ungar, M. (2008). Resilience across cultures. British Journal of Social Work, 38, 218-235. doi:10.1093/bjsw/bcl343

Vicary, J. R., Smith, E. A., Swisher, J. D., Hopkins, A. M., Elek, E., Bechtel , L. J., \& Henry, K. L. (2006). Results of a 3-year study of two methods of delivery of life skills training. Health Education \& Behavior, 33(3), 325-339. doi:10.1177/1090198105285020
World Health Organization. (1997). Life skills education for children and adolescents in schools. Geneva, Switzerland: Programme on Mental Health World Health Organization.

Yunes, M. A. M. (2003). Psicologia positiva e resiliência: O foco no indivíduo e na família. Psicologia em Estudo, 8, 75-84.
Recebido: 14/10/2013

$1^{a}$ revisão: $03 / 12 / 2013$

$2^{a}$ revisão: $14 / 02 / 2014$

Aceite final: 13/03/2014 